\title{
Inhibition of Inwardly Rectifying Potassium (Kir) 4.1 Channels Facilitates Brain-Derived Neurotrophic Factor (BDNF) Expression in Astrocytes
}

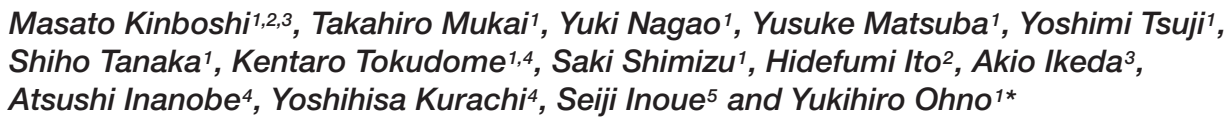

1 Laboratory of Pharmacology, Osaka University of Pharmaceutical Sciences, Osaka, Japan, ${ }^{2}$ Department of Neurology, Wakayama Medical University, Wakayama, Japan, ${ }^{3}$ Department of Epilepsy, Movement Disorders and Physiology, Graduate School of Medicine, Kyoto University, Kyoto, Japan, ${ }^{4}$ Department of Molecular and Cellular Pharmacology, Graduate School of Medicine, Osaka University, Osaka, Japan, ${ }^{5}$ Education and Research Center for Fundamental Pharmaceutical Sciences, Osaka University of Pharmaceutical Sciences, Osaka, Japan
OPEN ACCESS

Edited by: Bernard Attali, Tel Aviv University, Israel

Reviewed by: Maria Cristina D'Adamo, University of Malta, Malta

Petra Wahle,

Ruhr University Bochum, Germany

*Correspondence:

Yukihiro Ohno

yohno@gly.oups.ac.jp

Received: 17 August 2017 Accepted: 24 November 2017 Published: 07 December 2017

Citation:

Kinboshi M, Mukai T, Nagao $Y$, Matsuba Y, Tsuji Y, Tanaka S, Tokudome K, Shimizu S, Ito $H$,

Ikeda A, Inanobe A, Kurachi Y, Inoue $S$ and Ohno Y (2017) Inhibition of Inwardly Rectifying Potassium (Kir) 4.1 Channels Facilitates Brain-Derived Neurotrophic Factor (BDNF) Expression in Astrocytes.

Front. Mol. Neurosci. 10:408. doi: 10.3389/fnmol.2017.00408
Inwardly rectifying potassium (Kir) 4.1 channels in astrocytes regulate neuronal excitability by mediating spatial potassium buffering. Although dysfunction of astrocytic Kir4.1 channels is implicated in the development of epileptic seizures, the functional mechanisms of Kir4.1 channels in modulating epileptogenesis remain unknown. We herein evaluated the effects of Kir4.1 inhibition (blockade and knockdown) on expression of brain-derived neurotrophic factor (BDNF), a key modulator of epileptogenesis, in the primary cultures of mouse astrocytes. For blockade of Kir4.1 channels, we tested several antidepressant agents which reportedly bound to and blocked Kir4.1 channels in a subunit-specific manner. Treatment of astrocytes with fluoxetine enhanced BDNF mRNA expression in a concentration-dependent manner and increased the BDNF protein level. Other antidepressants (e.g., sertraline and imipramine) also increased the expression of BDNF mRNA with relative potencies similar to those for inhibition of Kir4.1 channels. In addition, suppression of Kir4.1 expression by the transfection of small interfering RNA (siRNA) targeting Kir4.1 significantly increased the mRNA and protein levels of BDNF. The BDNF induction by Kir4.1 siRNA transfection was suppressed by the MEK1/2 inhibitor U0126, but not by the p38 MAPK inhibitor SB202190 or the JNK inhibitor SP600125. The present results demonstrated that inhibition of Kir4.1 channels facilitates BDNF expression in astrocytes primarily by activating the Ras/Raf/MEK/ERK pathway, which may be linked to the development of epilepsy and other neuropsychiatric disorders.

Keywords: astrocytes, Kir4.1 channels, BDNF, epilepsy, antidepressants 


\section{INTRODUCTION}

Astrocytes are the major cell component of glial cells and play crucial roles in regulation of neural activity in the brain. By forming tripartite synapses in conjunction with presynaptic and postsynaptic neural components, astrocytes regulate ion and water homeostasis, metabolize neurotransmitters (e.g., glutamate, GABA, and glycine), and secrete various neuroactive substances including gliotransmitters, neurotrophic factors and cytokines (Araque et al., 1999; Parpura and Zorec, 2010; Devinsky et al., 2013). Among the diverse functions of astrocytes, spatial potassium buffering is a key mechanism for the maintenance of neural activity, which removes excessive extracellular $\mathrm{K}^{+}$ secreted from excited neurons (Walz, 2000; Kofuji and Newman, 2004; Simard and Nedergaard, 2004; Ohno et al., 2015). The potassium buffering currents are primarily mediated by the inwardly rectifying potassium (Kir) channels containing the Kir4.1 subunit, Kir4.1 channels (homo-tetramer of Kir4.1) and Kir4.1/5.1 channels (hetero-tetramer of Kir4.1 and Kir5.1), in astrocytes (Neusch et al., 2006; Ohno et al., 2015). In addition, spatial potassium buffering is known to be linked to glutamate and water uptake by astrocytes (Olsen and Sontheimer, 2008).

The Kir4.1 knockout animals showed severe motor impairment (e.g., ataxia and tremor), epileptic symptoms (e.g., jerky movements and convulsive seizures) and early mortality (within 3 weeks after birth) (Kofuji et al., 2000; Neusch et al., 2001; Djukic et al., 2007). In addition, Kir4.1 expression in astrocytes has been shown to be reduced (down-regulated) in the brain regions related to seizure foci in animal models of epilepsy (Ferraro et al., 2004; Inyushin et al., 2010; Harada et al., 2013). These findings suggest that dysfunction of astrocytic Kir4.1 channels can cause seizure generation probably by disrupting spatial potassium buffering. Furthermore, emerging evidence shows that loss-of-function mutations in the human KCNJ10 gene encoding Kir4.1 cause the epileptic disorders called "EAST" (Epilepsy, Ataxia, Sensorineural deafness and Tubulopathy) or "SeSAME" (Seizures, Sensorineural deafness, Ataxia, Mental retardation, and Electrolyte imbalance) syndrome (Bockenhauer et al., 2009; Scholl et al., 2009; Reichold et al., 2010). Patients with EAST/SeSAME syndrome manifested a seizure phenotype characterized by generalized tonic-clonic seizures (GTCSs) with the initial appearance within a few months after birth. Moreover, association of single nucleotide polymorphisms (SNPs) of KCNJ10 with temporal lobe epilepsy (TLE) accompanying febrile seizures (Heuser et al., 2010) and down-regulation of the Kir4.1 expression in seizure focus specimens from TLE patients (Das et al., 2012; Heuser et al., 2012; Steinhäuser et al., 2012) were also reported. Thus, dysfunction of Kir4.1 channels in astrocytes seems to be involved in the pathogenesis of human epileptic disorders. However, the pathophysiological mechanisms of astrocytic Kir4.1 channels in modulating epileptogenesis (development of chronic epilepsy) are still unknown.

Astrocytes release a variety of neuroactive molecules such as gliotransmitters (e.g., glutamate, ATP, and D-serine), neurotrophic factors [e.g., nerve growth factor (NGF), brain-derived neurotrophic factor (BDNF), and glia-derived neurotrophic factor (GDNF)], and cytokines [e.g., tumor necrosis factor- $\alpha$ (TNF- $\alpha$ ) and interleukin-1 $\beta$ (IL-1 $\beta$ )] (Parpura and Zorec, 2010; Devinsky et al., 2013). Among them, BDNF is a key modulator of epileptogenesis, which influences on synaptic plasticity, neural sprouting, neurogenesis, and reactive gliosis (Kokaia et al., 1995; Jankowsky and Patterson, 2001; Hagihara et al., 2005; Jean et al., 2008; Quesseveur et al., 2013). Specifically, the mRNA and protein levels of BDNF are known to be elevated in various animal models of epilepsy and in human epileptic brains (Jankowsky and Patterson, 2001). In addition, genetic knockdown of BDNF has been shown to suppress the development of epilepsy (Kokaia et al., 1995; Barton and Shannon, 2005; Heinrich et al., 2011; Grabenstatter et al., 2012). Inhibition of the BDNF receptor TrkB has also been shown to prevent kindling epileptogenesis in TLE models and ameliorate status epilepticus (SE)-induced chronic recurrent seizures (Binder et al., 1999; Liu et al., 2013). Moreover, astrocytes express not only full-length TrkB, but also truncated TrkB receptors (Rose et al., 2003; Cheng et al., 2007), and the truncated TrkB, especially truncated TrkB-T1 receptors have been reported to facilitate astrogliosis, which may be involved in epileptogenesis (Goutan et al., 1998; Climent et al., 2000). All these evidences illustrate a causal role of BDNF in the development of epilepsy.

In the present study, we evaluated the effects of inhibition (blockade and knockdown) of Kir4.1 channels on the expression of BDNF and other neurotrophic factors in primary cultures of astrocytes to explore the mechanism of Kir4.1 channels in regulating epileptogenesis. For blockade of Kir4.1 channels, we tested several antidepressants agents (e.g., fluoxetine), which reportedly blocked Kir4.1 channels in a subunit-specific manner (Ohno et al., 2007; Su et al., 2007; Furutani et al., 2009; Ohno et al., 2015). In addition, small interfering RNA (siRNA) targeting Kir4.1 was employed to knockdown the Kir4.1 expression. The present results demonstrated for the first time that inhibition of Kir4.1 channels facilitates the expression of BDNF in astrocytes, which may be linked to the development of epilepsy and other neuropsychiatric disorders.

\section{MATERIALS AND METHODS}

\section{Astrocyte Primary Cultures}

Primary cultures of cerebrocortical astrocytes were prepared from 1 or 2 day-old new born ICR mice (Japan SLC, Shizuoka, Japan). All the protocols of experiments were approved by the Animal Research Committee at Osaka University of Pharmaceutical Sciences. After brains were removed from the skulls under isoflurane anesthesia, meninges were excised carefully and forebrains were dissected into small pieces. Cells were dissociated with $0.25 \%$ trypsin (Sigma-Aldrich, St. Louis, MO, United States), plated to $25 \mathrm{~cm}^{2}$ flasks with Dulbecco's Modified Eagle Medium (DMEM; Nacalai Tesque, Kyoto, Japan) containing $10 \%$ heat-inactivated fetal bovine serum (FBS; biowest, Nuaillé, France), and incubated at $37^{\circ} \mathrm{C}$ with $5 \% \mathrm{CO}_{2} / 95 \%$ air. After the cells reached confluence (7 days), adherent cells were shaken by hand, detached with $0.05 \%$ trypsin, and plated to $75 \mathrm{~cm}^{2}$ flasks. After three times passage (21 days), the cells were plated to 24-well plates for mRNA analysis or 
$6 \mathrm{~cm}$ diameter dishes for protein analysis, and, thereafter, culture medium was replaced with serum-free DMEM. The cells were then subjected to the experiments under serum-free condition after 10 days or more.

\section{Immunofluorescence Staining}

Astrocytes plated on glass coverslips were fixed in $4 \%$ paraformaldehyde, and then blocked in $0.3 \%$ Triton X-100 in phosphate buffered saline containing $1 \%$ bovine serum albumin (BSA; Wako, Osaka, Japan) for $30 \mathrm{~min}$. After blocking, astrocytes were incubated with primary antibodies for glial fibrillary acidic protein (GFAP: a specific marker for astrocytes) (mouse monoclonal, 1:200; Progen, Heidelberg, Germany, RRID: AB_1541328), Kir4.1 (rabbit polyclonal, 1:200; Alomone Labs, Jerusalem, Israel, RRID: AB_2040120), BDNF (goat polyclonal, 1:200; Santa Cruz Biotechnology, Dallas, TX, United States, RRID: AB_2227954), and neuronal nuclear antigen (NeuN: a specific marker for neurons) (mouse monoclonal, 1:500; Millipore, Billerica, MA, United States, RRID: AB_2298772) at $4^{\circ} \mathrm{C}$ overnight. Subsequently, secondary antibodies of tetramethylrhodamine-5- (and 6)-isothiocyanate (TRITC; red fluorescence) goat anti-mouse (1:200; Sigma-Aldrich, RRID: AB_261699), fluorescein isothiocyanate (FITC; green fluorescence) goat anti-rabbit (1:200; Sigma-Aldrich, RRID: AB_259384), Alexa Fluor546 (red fluorescence) donkey anti-mouse (1:200; Thermo Fisher scientific, Waltham, MA, United States, RRID: AB_2534012), Alexa Fluor488 (green fluorescence) donkey anti-goat (1:200; Thermo Fisher scientific, RRID: AB_2534102), or Alexa Fluor647 (blue fluorescence) donkey anti-rabbit (1:200; Thermo Fisher scientific, RRID: AB_2536183) were, respectively, used for visualization. Immunofluorescence images were obtained with a confocal laser scanning microscope (Carl Zeiss Japan, LSM 700 ZEN, Tokyo, Japan).

\section{Quantitative Reverse Transcription (RT)-PCR}

Total RNA was extracted from astrocytes in each well of a 24-well plate using ISOGEN ${ }^{\circledR}$ (Nippon Gene, Tokyo, Japan) according to the manufacturer's instructions. Purified total RNA was used to synthesize cDNA with a PrimeScript RT reagent Kit (Takara Bio, Shiga, Japan) for $15 \mathrm{~min}$ at $37^{\circ} \mathrm{C}$ and for $5 \mathrm{~s}$ at $85^{\circ} \mathrm{C}$. cDNA was amplified in a PCR reaction containing SYBR ${ }^{\circledR}$ Premix Ex Taq ${ }^{\circledR}$ (Takara Bio) and $0.4 \mu \mathrm{M}$ of each gene-specific primer (Table 1). The reactions were performed in a Thermal Cycler Dice Real Time System Single MRQ (Takara Bio; TP870) with a PCR profile comprising an initial denaturation step for $2 \mathrm{~min}$ at $95^{\circ} \mathrm{C}, 40$ cycles with $5 \mathrm{~s}$ at $95^{\circ} \mathrm{C}$ and $60 \mathrm{~s}$ at $60^{\circ} \mathrm{C}$, and a final step for $15 \mathrm{~s}$ at $95^{\circ} \mathrm{C}, 30 \mathrm{~s}$ at $60^{\circ} \mathrm{C}$, and $15 \mathrm{~s}$ at $95^{\circ} \mathrm{C}$. Following amplification, reaction specificity for each PCR run was confirmed by melt curve analysis.

\section{BDNF Enzyme-Linked Immunosorbent Assay (ELISA)}

A quantitative measure of BDNF concentration in each cell lysate was performed with a BDNF $\mathrm{E}_{\max }{ }^{\otimes}$ ImmunoAssay
System (Promega, Madison, WI, United States) according to the manufacturer's instructions. As an internal control, total protein was measured in the lysates for use in normalizing results. The BDNF levels were expressed as a ratio to total protein.

\section{Kir4.1 siRNA Transfection}

The sequences of siRNA targeting mouse Kir4.1 were designed as shown in Table 1 (RNAi Co., Tokyo, Japan). Astrocytes were transfected with siRNA or a negative control using Lipofectamine $^{\circledast}$ RNAiMAX Transfection reagent (Thermo Fisher scientific) according to the manufacturer's instructions. After $6 \mathrm{~h}$ of treatment with siRNA, the medium was replaced and incubated at $37^{\circ} \mathrm{C}$ with $5 \% \mathrm{CO}_{2} / 95 \%$ air until collection.

\section{Western Blotting}

Western blotting was performed as published previously (Ohno et al., 2009; Harada et al., 2013; Nagao et al., 2013). Briefly, cell lysates were incubated with sodium dodecyl sulfate-polyacrylamide gel electrophoresis (SDS-PAGE) sample buffer for $5 \mathrm{~min}$ at $95^{\circ} \mathrm{C}$. Then, each sample ( $5 \mu \mathrm{g} / \mathrm{lane}$ ) was run on a $15 \%$ polyacrylamide gel and transferred to a PVDF membrane (GE Healthcare, Buckinghamshire, United Kingdom). The membrane was blocked with TBS-T (25 mM Tris, $150 \mathrm{mM}$ $\mathrm{NaCl}$, and $0.1 \%$ Tween 20 ) containing $5 \%$ skimmed milk, and incubated with the primary antibodies for Kir4.1 (rabbit polyclonal, 1:500) or $\beta$-actin (mouse monoclonal, 1:1000; Sigma-Aldrich, RRID: AB_476743) at $4^{\circ} \mathrm{C}$ overnight. Then, the membrane was incubated with horseradish peroxidase (HRP)-conjugated secondary antibodies, goat anti-rabbit IgG-HRP conjugated (1:2000; Santa Cruz Biotechnology, RRID: AB_631746) or sheep anti-mouse IgG-HRP conjugated (1:2000; GE Healthcare, RRID: AB_772210) for $60 \mathrm{~min}$ at room temperature. Specific bands were detected with the enhanced chemiluminescence methodology (Amersham ECL Western blotting detection reagents and analysis system, GE Healthcare) by a lumino imaging analyzer (LAS-3000, FUJIFILM, Tokyo, Japan). To normalize for protein loading, the band intensity in each lane was standardized to the intensity of the $\beta$-actin band in the same lane after stripping and reprobing processes.

\section{Electrophysiology}

Whole-cell patch clamp electrophysiology was performed as described previously (Ohno et al., 2007; Su et al., 2007). Briefly, human Kir4.1 inserted into IRES vectors (pIRES2-DsRed2, Clontech, Mountain view, CA, United States) were transfected into HEK293T cells using Fugene6 transfectant (Roche Diagnostics, Tokyo, Japan). The transfected cells were continuously perfused with a bathing solution (in $\mathrm{mM}$; $112 \mathrm{NaCl}, 30 \mathrm{KCl}, 5 \mathrm{HEPES}, 2 \mathrm{CaCl}_{2}, 0.53 \mathrm{MgCl}_{2}$, and 5.5 glucose, $\mathrm{pH} 7.4$ ) at a flow rate of $1-1.5 \mathrm{ml} / \mathrm{min}$. The recording micropipettes had input resistances of 1.4-1.8 $\mathrm{M} \Omega$ when filled with an internal solution containing (in $\mathrm{mM}$ ) $140 \mathrm{KCl}, 2 \mathrm{MgCl}_{2}$, 5 EGTA, and 5 HEPES (adjusted to pH7.25 with $\mathrm{KOH}$ ). The cells were voltage-clamped at $E_{K}$ (i.e., $-40 \mathrm{mV}$ in $30 \mathrm{mM}$ 
TABLE 1 | Sequences of siRNA and primers for quantitative reverse transcription (RT)-PCR.

\begin{tabular}{|c|c|c|}
\hline & Sense strand $\left(5^{\prime}-3^{\prime}\right)$ & Antisense strand $\left(5^{\prime}-3^{\prime}\right)$ \\
\hline \multicolumn{3}{|c|}{ Sequences of siRNA } \\
\hline \multirow[t]{2}{*}{ Kir4.1 } & GCCAAGUUCGCACttcctacc & taggaaGUGCGAACUUGGCAG \\
\hline & Forward primer $\left(5^{\prime}-3^{\prime}\right)$ & Reverse primer $\left(5^{\prime}-3^{\prime}\right)$ \\
\hline \multicolumn{3}{|c|}{ Sequences of primers for RT-PCR } \\
\hline GAPDH & TGTGTCCGTCGTGGATCTGA & TTGCTGTTGAAGTCGCAGGAG \\
\hline Kir4.1 & TGAGGCAATGCTGAGAGGAG & CCCGTAGGCAGAAAAGAGGA \\
\hline BDNF & TCAAGTTGGAAGCCTGAATGAATG & CTGATGCTCAGGAACCCAGGA \\
\hline GDNF & TCAGCTGCCCAGCACATTTC & TGGGAGCATCAGCTACCACATC \\
\hline CNTF & GCTCACTTGTITCCTGGGACAGT & CCATCCACTGAGTCAAGGCTGAT \\
\hline NGF & TGCCAAGGACGCAGCПTC & TGAAGTITAGTCCAGTGGGCTTCAG \\
\hline Slc6a4 & TGGCTACATGGCTGAGATGAGG & AAGAATGTGGATGCTGGCATGTTA \\
\hline
\end{tabular}

$\left.\left[\mathrm{K}^{+}\right]_{\mathrm{o}}\right)$ and stepped to depolarized or hyperpolarized potentials (e.g., $\pm 70 \mathrm{mV}$ ) from $\mathrm{E}_{\mathrm{K}}$. The clamp voltage and associated macroscopic currents were recorded with an Axopatch 200B amplifier (Axon Instruments, Union City, CA, United States), fed through a digitizer (Digidata 1322A, Axon Instruments) and stored in a computer with the data acquisition system Clampex 9.2 (Axon Instruments). Test drugs were dissolved in bathing solution and perfused until the response reached a plateau (usually $5 \mathrm{~min}$ ). All experiments were performed at room temperature. In each cell, an excessive concentration ( $3 \mathrm{mM}$ ) of $\mathrm{Ba}^{2+}$ which totally blocked Kir channels was applied at the end of each experiment. The Kir channel currents were measured by subtracting the $\mathrm{Ba}^{2+}$-resistant currents from the total current. The response to the test drug was estimated as the current ratio $\left(\mathrm{I}_{\text {Drug }} / \mathrm{I}_{\text {Control }}\right.$ ) at $-110 \mathrm{mV}$, which was obtained by dividing the current recorded in the presence of the drug with the equivalent current recorded in the absence of the drug.

\section{Drugs}

Sertraline hydrochloride, U0126, and SB202190 were purchased from Wako Pure Chemicals Industries (Osaka, Japan). Fluoxetine hydrochloride was purchased from LKT Labs (St. Paul, MN, United States). Imipramine hydrochloride and mianserin hydrochloride were purchased from Sigma-Aldrich. Fluvoxamine maleate and SP600125 were purchased from Tokyo Chemical Industries (Tokyo, Japan). Other common laboratory reagents were also obtained from commercial sources.

\section{Statistical Analysis}

All data were expressed as the mean \pm SEM. Comparisons between two groups were performed by Mann-Whitney test (mRNA levels or protein levels in astrocytes transfected with siRNA or exposed to each drug). Statistical significance of differences among multiple groups was determined by Kruskal-Wallis followed by Steel-Dwass post hoc test (mRNA levels in astrocytes exposed to multiple drugs or a certain drug at multiple concentrations). A $P$-value of less than 0.05 was considered statistically significant.

\section{RESULTS}

\section{Expression Patterns of Kir4.1 and BDNF in Cultured Astrocytes}

We first confirmed the expression pattern of Kir4.1 and BDNF in our astrocyte primary cultures using the immunofluorescent double staining method. Confocal laser microscopic analysis of single astrocyte revealed that Kir4.1-immnoreactivity (IR) was mostly detected in both somata and foot processes probed by the astrocyte marker GFAP (Figure 1Aa), resembling in vivo expression patterns reported previously (Harada et al., 2013; Nagao et al., 2013). A lot of granules positive for BDNF-IR, spreading from somata to end foots, were also co-stained with GFAP (Figure 1Ab). In addition, the BDNF-IR were detected in Kir4.1-IR positive cells (Figure 1Ac). In the confluent conditions, astrocytes with various cell shapes normally gathered together and formed large cell clusters (Figure 1B). These astrocytes possess numerous GFAP-IR negative thin processes surrounding the GFAP-IR positive somata. Interestingly, BDNF were densely expressed in these fine processes of astrocytes, reflecting a secretary process of BDNF. On the other hand, our astrocyte primary cultures did not contain NeuN (a neuron-specific marker)-IR positive cells (data not shown).

\section{Effects of Kir4.1 Channel Blocker on BDNF Expression in Astrocytes}

To evaluate expressional changes in BDNF due to the Kir4.1 channel blockade, cultured astrocytes were treated with a subunit-specific Kir4.1 channel blocker, fluoxetine (see Supplementary Figure S1) for $8 \mathrm{~h}$. Under these conditions, fluoxetine $(1-30 \mu \mathrm{M})$ increased BDNF mRNA expression in a concentration-dependent manner and this increase reached statistical significance at 3-30 $\mu \mathrm{M}$ (Figure 2A). In addition, the BDNF protein levels also significantly increased in astrocytes following the treatment with $30 \mu \mathrm{M}$ fluoxetine for $24 \mathrm{~h}$ (Figure 2B).

We also tested several antidepressants which exhibit different blocking activities for Kir4.1 channels. When selective serotonin reuptake inhibitors (SSRIs), fluoxetine $(30 \mu \mathrm{M})$, sertraline 


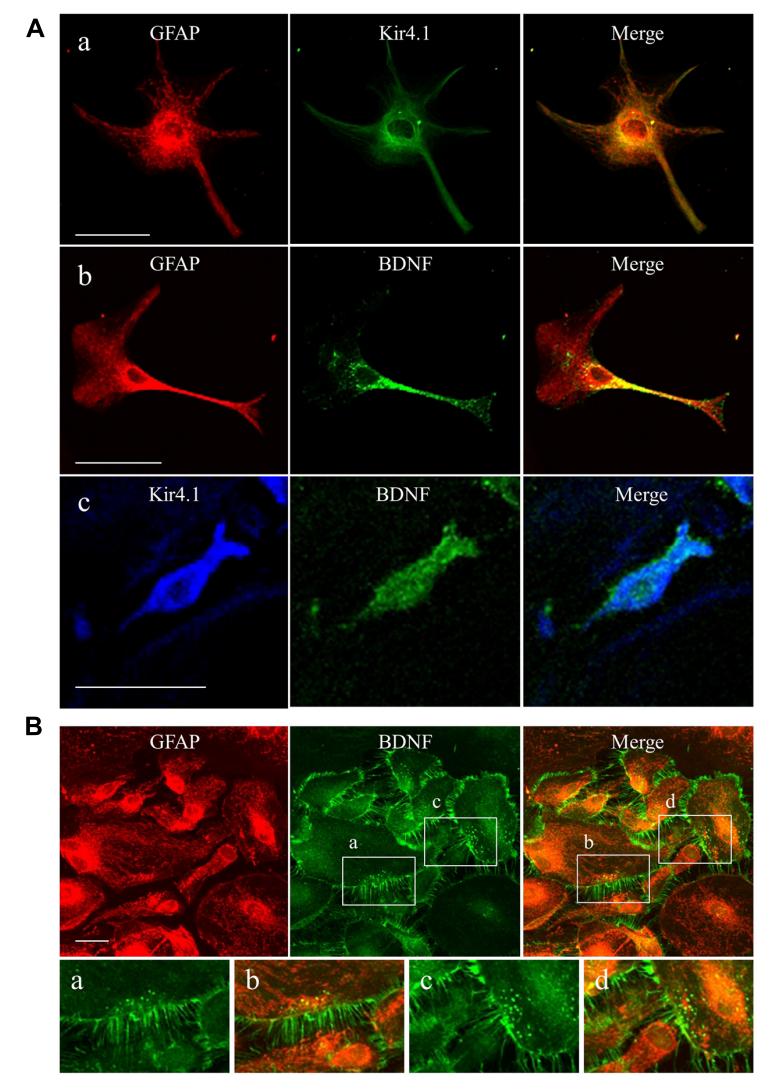

FIGURE 1 | Expression patterns of Kir4.1 channels and BDNF in cultured astrocytes. (A) Representative images of double-immunofluorescent staining for GFAP and Kir4.1 (a), GFAP and BDNF (b), or Kir4.1 and BDNF (c) in single astrocytes. (B) Clustered astrocytes stained for GFAP and BDNF in culture preparations. Lower panels showed magnified images of region indicated by squares (a-d). Scale bar: $50 \mu \mathrm{m}$ (Aa, Ab, and B) or $25 \mu \mathrm{m}$ (Ac)

$(30 \mu \mathrm{M})$ and fluvoxamine $(100 \mu \mathrm{M})$, a tricyclic antidepressant imipramine $(100 \mu \mathrm{M})$, and a tetracyclic antidepressant mianserin $(100 \mu \mathrm{M})$ were applied to the Kir4.1 channel-expressing HEK293 cells, these agents inhibited Kir4.1-conducted potassium currents in the following order, sertraline $>$ fluoxetine $>$ imipramine $>>$ fluvoxamine $>>$ mianserin (Figure 3A). Treatment of cultured astrocytes with these agents (at $30 \mu \mathrm{M}$ ) for $8 \mathrm{~h}$ increased the BDNF mRNA expression with potencies as follows, sertraline $>$ fluoxetine $>$ imipramine $>>$ fluvoxamine $=$ mianserin (Figure 3B). Thus, there was a similarity in the relative potencies of antidepressants for the blockade of Kir4.1 channels and for the induction of BDNF mRNA in astrocytes. These drugs did not affect the cell viability of astrocytes (data not shown). In addition, it should be noted that mRNA expression of the serotonin transporter (Slc6a4) was not detected in our astrocyte preparations (data not shown).

\section{Effects of Kir4.1 Knockdown on BDNF Expression in Astrocytes}

To knockdown the Kir4.1 expression, astrocytes were transfected with Kir4.1 siRNA (Table 1), and total RNA or protein was
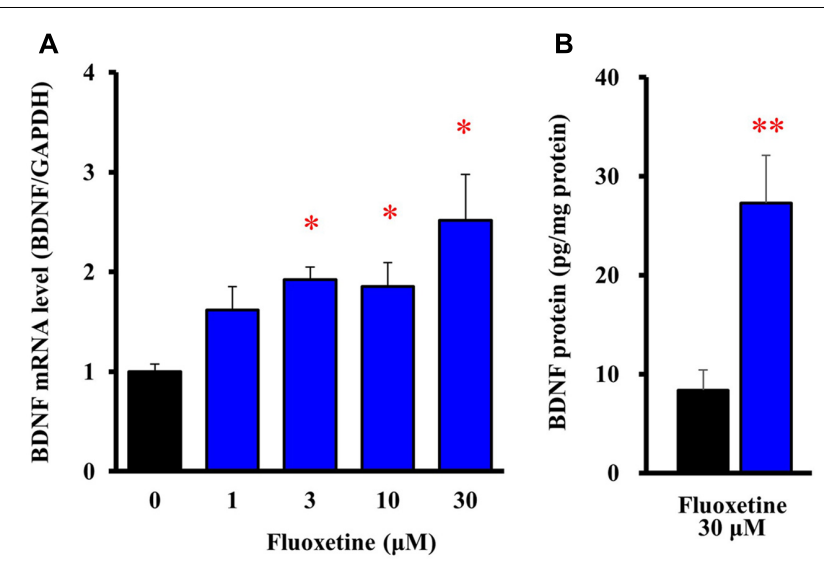

FIGURE 2 | Effects of fluoxetine on BDNF expression in astrocytes. (A) Effects of fluoxetine on BDNF mRNA expression. Astrocytes were treated with the indicated concentrations of fluoxetine (1-30 $\mu \mathrm{M})$ and BDNF mRNA expression was analyzed by quantitative reverse transcription (RT)-PCR. (B) Effects of fluoxetine on BDNF protein expression. Astrocytes treated with $30 \mu \mathrm{M}$ fluoxetine for $24 \mathrm{~h}$ and the BDNF protein levels were analyzed by ELISA. The BDNF mRNA levels are expressed as the ratio to GAPDH mRNA. Each point represents the mean \pm SEM of $5-6$ separate experiments. ${ }^{*} P<0.05$,

$* * P<0.01$, significantly different from the astrocytes treated with vehicle.

isolated at 24 or $48 \mathrm{~h}$ after the transfection. The results of quantitative reverse transcription (RT)-PCR showed that the Kir4.1 siRNA effectively suppressed the Kir4.1 expression in astrocytes, compared with the negative control, from 24 to $48 \mathrm{~h}$ after the transfection (Figure 4A). In addition, Western blotting confirmed that the Kir4.1 protein level was significantly decreased at $24 \mathrm{~h}$ after the transfection, more markedly at $48 \mathrm{~h}$ (Figure 4B).

In astrocytes transfected with Kir4.1 siRNA, mRNA expression of BDNF was significantly increased at both 24 and $48 \mathrm{~h}$ after the transfection (Figure 5A). The BDNF protein levels were also increased by Kir4.1 knockdown at 24 and 48 h, and a statistical significance was obtained at $48 \mathrm{~h}$ (Figure 5B).

We also examined the effects of Kir4.1 knockdown on mRNA expression of other neurotrophic factors, GDNF, ciliary neurotrophic factor (CNTF), and NGF. Kir4.1 knockdown slightly, but significantly, increased GDNF and CNTF mRNA at $48 \mathrm{~h}$. However, the mRNA expression of neither GDNF (24 h), CNTF (24 h) nor NGF was affected by Kir4.1 knockdown (Figure 5C).

\section{Signaling Pathways Regulating BDNF Expression by Kir4.1 Knockdown}

To clarify the signaling pathways underlying the BDNF expression changes due to Kir4.1 knockdown, astrocytes were pretreated with U0126 [a non-competitive inhibitor of mitogenactivated protein kinase (MEK) 1/2, $10 \mu \mathrm{M}$ ], SB202190 (an inhibitor of p38 MAPK, $10 \mu \mathrm{M}$ ), or SP600125 [an inhibitor of Jun amino-terminal kinase (JNK), $10 \mu \mathrm{M}$ ] for $30 \mathrm{~min}$ before the transfection with Kir4.1 siRNA or a negative control. These agents did not affect the Kir4.1 siRNA-induced knockdown of Kir4.1 in transfected astrocytes (Figure 6A). However, U0126 

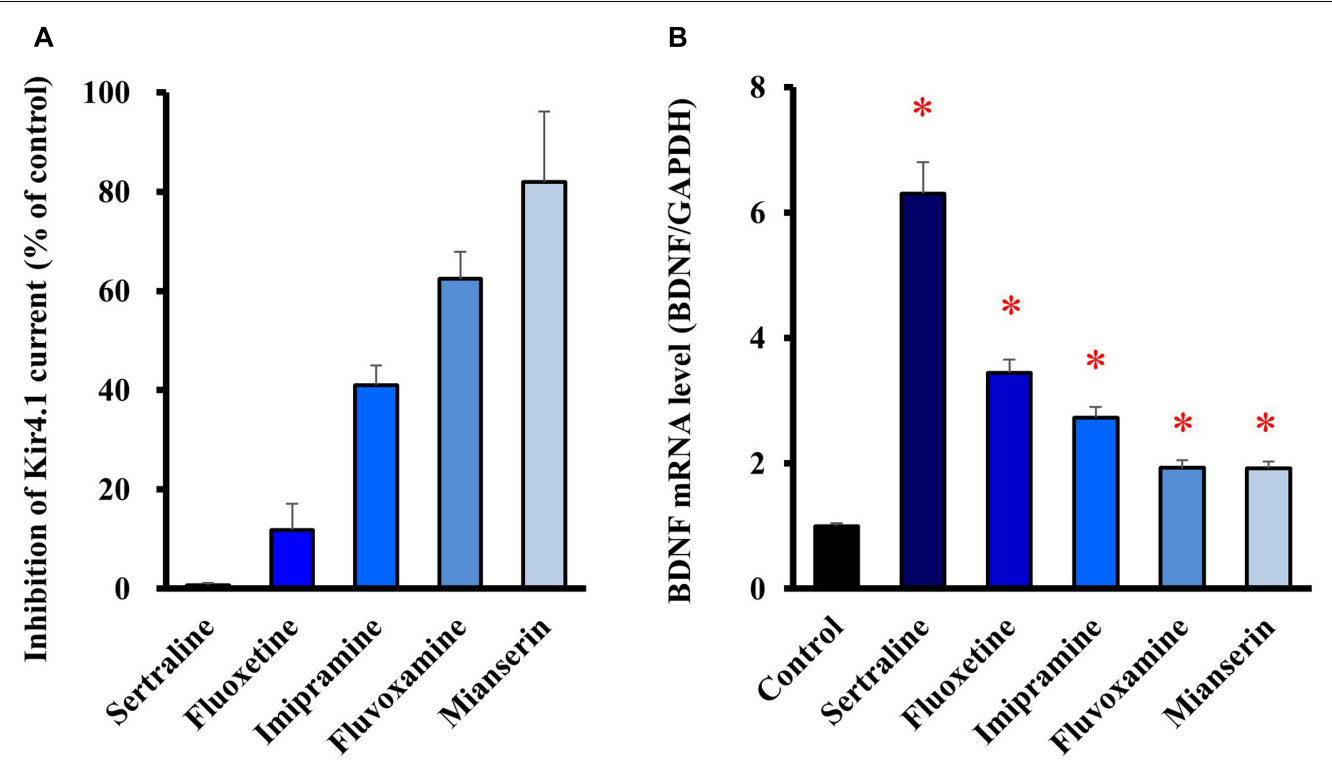

FIGURE 3 | Relationship between potencies of antidepressants for the blockade of Kir4.1 channels and for the astrocytic BDNF induction. (A) Inhibition percentage (IDrug $/$ I $_{\text {Control }}$ ) of Kir4.1-conducted currents by sertraline (30 $\left.\mu \mathrm{M}\right)$, fluoxetine (30 $\left.\mu \mathrm{M}\right)$, imipramine (100 $\left.\mu \mathrm{M}\right)$, fluvoxamine (100 $\left.\mu \mathrm{M}\right)$, or mianserin (100 $\left.\mu \mathrm{M}\right)$ in HEK293T cells. (B) Enhancement of BDNF mRNA expression by sertraline, fluoxetine, imipramine, fluvoxamine, or mianserin in astrocytes. Astrocytes were treated with each antidepressant $(30 \mu \mathrm{M})$ for $8 \mathrm{~h}$ and the BDNF mRNA expression was analyzed by RT-PCR. The BDNF mRNA levels are expressed as the ratio to GAPDH mRNA. Each point represents the mean \pm SEM of six separate experiments. ${ }^{*} P<0.05$, significantly different from the astrocytes treated with vehicle.
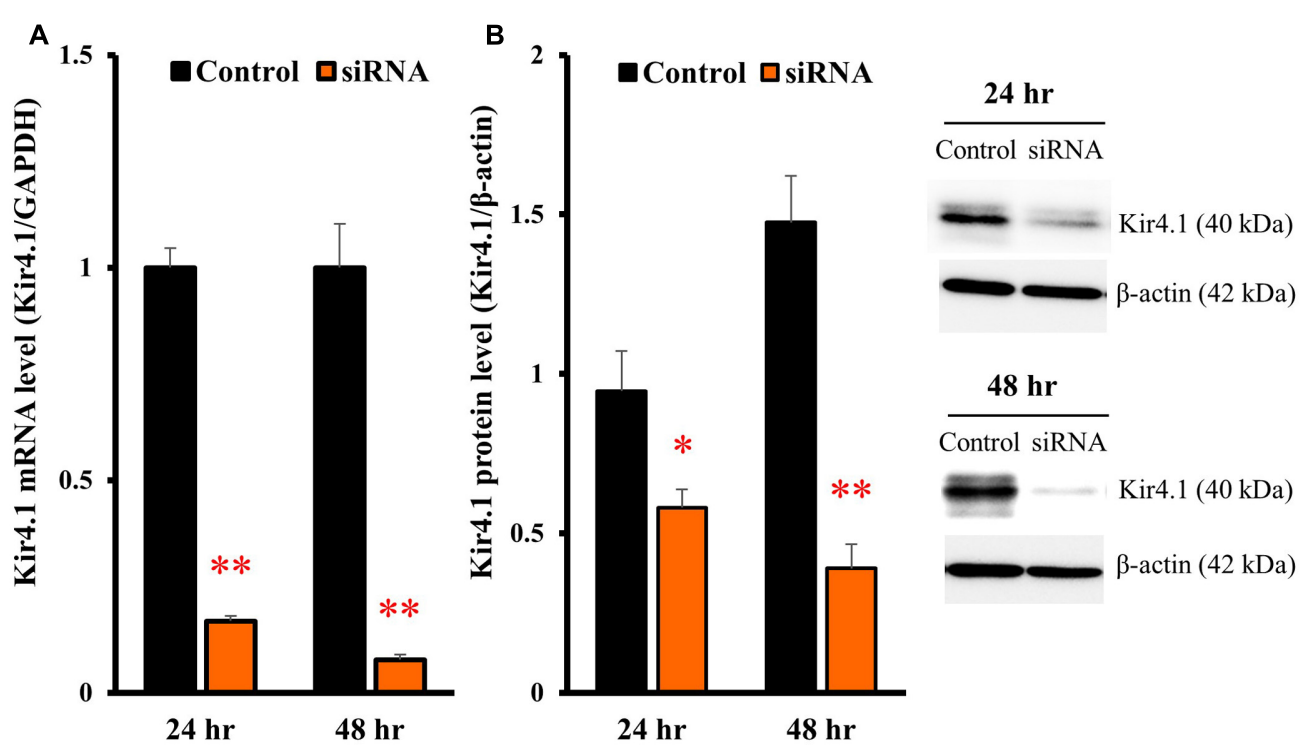

FIGURE 4 | Knockdown of Kir4.1 channels in astrocytes transfected with Kir4.1 siRNA. Kir4.1 mRNA levels (A) and protein levels (B) were analyzed by RT-PCR or Western blotting, respectively, at 24 or $48 \mathrm{~h}$ after transfection of Kir4.1 siRNA. The Kir4.1 mRNA and protein levels are expressed as the ratio to GAPDH mRNA and $\beta$-actin protein, respectively. Each point represents the mean \pm SEM of $5-6$ separate experiments. ${ }^{*} P<0.05,{ }^{* *} P<0.01$, significantly different from the negative control.

significantly suppressed the Kir4.1 siRNA-induced increase in BDNF mRNA expression (Figure 6B). In contrast, SB202190 or SP600125 did not fully inhibit the induction of BDNF mRNA expression due to Kir4.1 siRNA, whereas a slight reduction in BDNF mRNA level was observed with these agents.

\section{DISCUSSION}

Lack of a specific antagonist for Kir4.1 channels, as well as the early premature death of Kir4.1 knockout animals, have hampered the detailed analysis of Kir4.1 channel functions 

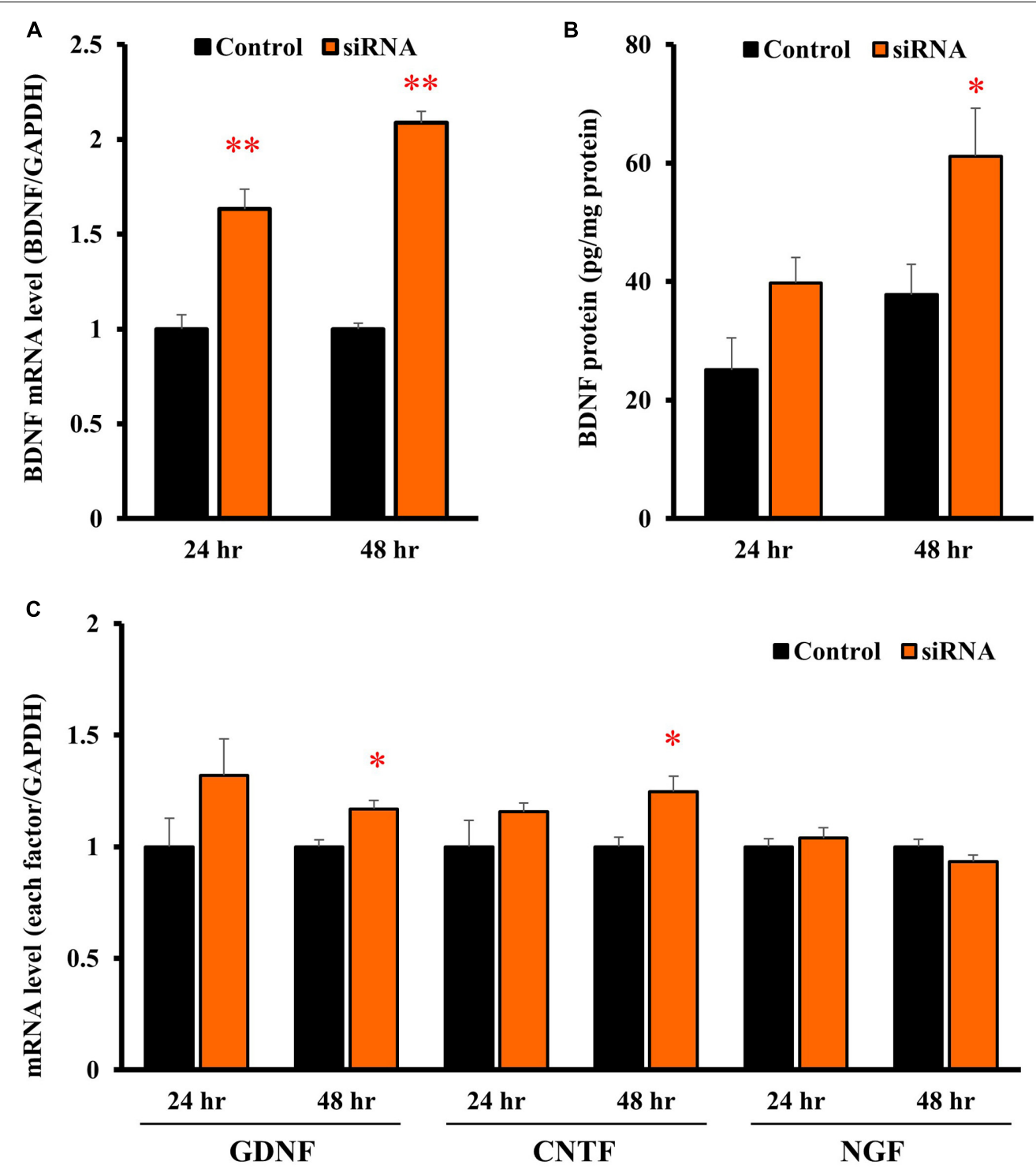

FIGURE 5 | Effects of Kir4.1 knockdown on BDNF and other neurotrophic factors in astrocytes. Effects of Kir4.1 siRNA transfection on BDNF mRNA (A) and protein levels (B). The BDNF mRNA levels and protein levels were analyzed by RT-PCR and ELISA, respectively, in astrocytes at 24 or $48 \mathrm{~h}$ after the transfection of Kir4.1 siRNA. (C) Effects of Kir4.1 siRNA transfection on mRNA levels of GDNF, CNTF, and NGF. The GDNF, CNTF, or NGF mRNA level was analyzed by RT-PCR in astrocytes at 24 or $48 \mathrm{~h}$ after transfection of Kir4.1 siRNA. The mRNA and protein levels are expressed as the ratio to GAPDH mRNA and $\beta$-actin protein, respectively. Each point represents the mean \pm SEM of $5-6$ separate experiments. ${ }^{*} P<0.05$, ${ }^{* *} P<0.01$, significantly different from the negative control.

for a long time. Nonetheless, we have previously shown that several antidepressant agents, including SSRIs and tricyclic antidepressants, specifically bound to and blocked Kir4.1 channels without affecting Kir1.1 or Kir2.1 channel activities (Ohno et al., 2007; Su et al., 2007; Furutani et al., 2009). These actions were not mediated by the serotonergic system (e.g., serotonin transporters), but were due to direct and specific binding to the pore region of Kir4.1 channels. Alanine-scan mutagenesis analysis revealed that two amino acids, Thr128 and Glu158, which are located on the pore and the 2nd transmembrane (TM-2) domain of Kir4.1, respectively, were responsible for the blockade of Kir4.1 channels by the antidepressants (e.g., fluoxetine and nortriptyline). In silico pharmacophore-docking analysis suggested that Thr128 and Glu158, facing the central cavity of Kir4.1 channels, bind to the antidepressants with hydrogen and ionic bonds, respectively (Furutani et al., 2009; Ohno et al., 2015). By testing these Kir4.1 channel blockers, we herein demonstrated that blockade of Kir4.1 channels significantly enhanced the BDNF production in astrocytes. Although we cannot directly translate the drug actions in Kir4.1-expressing HEK293 cells to those in astrocytes, various antidepressants increased BDNF mRNA expression with relative potencies (sertraline $>$ fluoxetine $>$ imipramine $>>$ fluvoxamine $=$ 

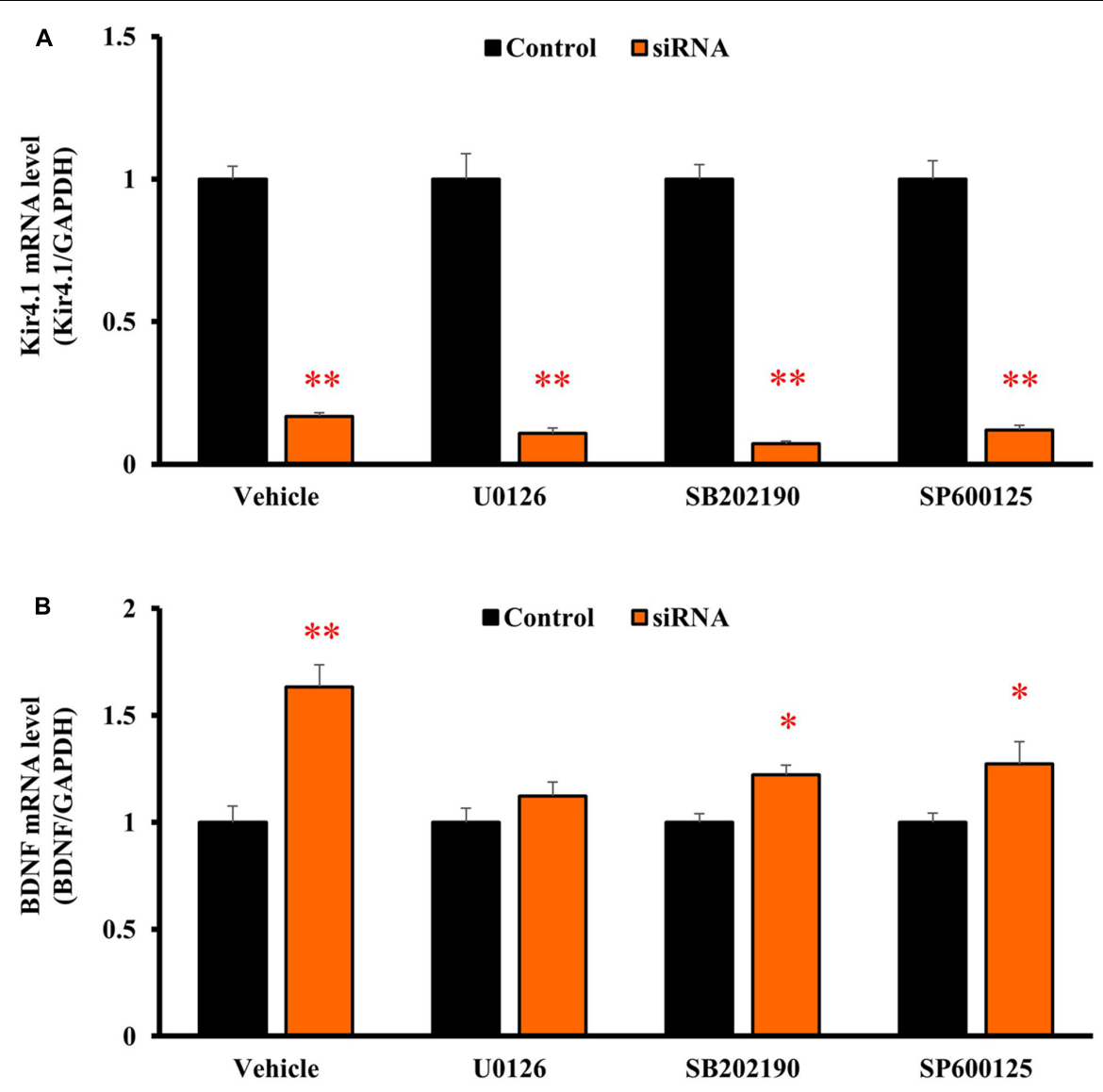

FIGURE 6 | Effects of U0126, SB202190, and SP600125 on BDNF mRNA induction by Kir4.1 knockdown in astrocytes. Astrocytes were pretreated with $10 \mu$ M U0126, $10 \mu$ M SB202190, or $10 \mu$ M SP600125 for 30 min, and transfected with Kir4.1 siRNA or a negative control. The mRNA expression of Kir4.1 (A) or BDNF (B) was analyzed by RT-PCR in astrocytes at $24 \mathrm{~h}$ after transfection of Kir4.1 siRNA. The mRNA levels are expressed as the ratio to GAPDH mRNA. Each point represents the mean \pm SEM of 5-6 separate experiments. $* P<0.05$, ${ }^{*} P<0.01$, significantly different from the negative control.

mianserin) consistent with those for the inhibition of Kir4.1 currents. It seems unlikely that antidepressants-induced BDNF expression is mediated by their interactions with serotonin transporters, since their relative potencies for Kir4.1 inhibition-induced BDNF expression were different from those for the inhibition of serotonin reuptake (imipramine $>$ fluoxetine $=$ fluvoxamine $>$ sertraline $>>$ mianserin) (Mortensen et al., 2001). Indeed, mRNA expression of serotonin transporter was not detected in our cultured astrocytes, consistent with previous studies (Kong et al., 2002; Li et al., 2012). Furthermore, suppression of Kir4.1 expression by the Kir4.1 siRNA transfection also enhanced BDNF production in astrocytes. The present results therefore strongly suggest that inhibition (blockade and expressional down-regulation) of Kir4.1 channels enhances BDNF synthesis and secretion from astrocytes. Interestingly, the modulatory role of Kir4.1 channels was relatively specific for the BDNF expression and much weakly affected the expression of other neurotrophic factors although the GDNF and CNTF expression were slightly increased.

Mechanisms for the BDNF induction by Kir4.1 channel inhibition are still uncertain at this stage. In the present study, the enhancement of BDNF mRNA expression by Kir4.1 knockdown was selectively suppressed by the MEK1/2 inhibitor U0126, but not by the p38 MAPK inhibitor SB202190 or the JNK inhibitor SP600125. These results suggest that Kir4.1 knockdown-induced BDNF expression in astrocytes is primarily mediated by activation of the Ras/Raf/MEK/ERK signaling pathways (Figure 7). This possibility was supported by the previous findings that the Ras/Raf/MEK/ERK signaling pathways regulate the transcription of $\mathrm{BDNF}$ and other survival/plasticity genes through interaction with cyclic AMP response element binding protein (CREB) (Curtis and Finkbeiner, 1999; Duman and Voleti, 2012). However, we cannot completely deny a possible involvement of the p38 MAPK and/or JNK system in the BDNF induction since SB202190 and SP600125 slightly reduced the enhancement of BDNF expression by Kir4.1 knockdown.

Evidence is accumulating that dysfunction of Kir4.1 channels causes epileptic disorders in humans. The mutation of the KCNJ10 gene encoding Kir4.1 conveys the EAST/SeSAME syndrome manifesting GTCS in the early postnatal stage (2-3 months after birth) (Bockenhauer et al., 2009; Scholl et al., 2009; Reichold et al., 2010). In addition, genetic association 


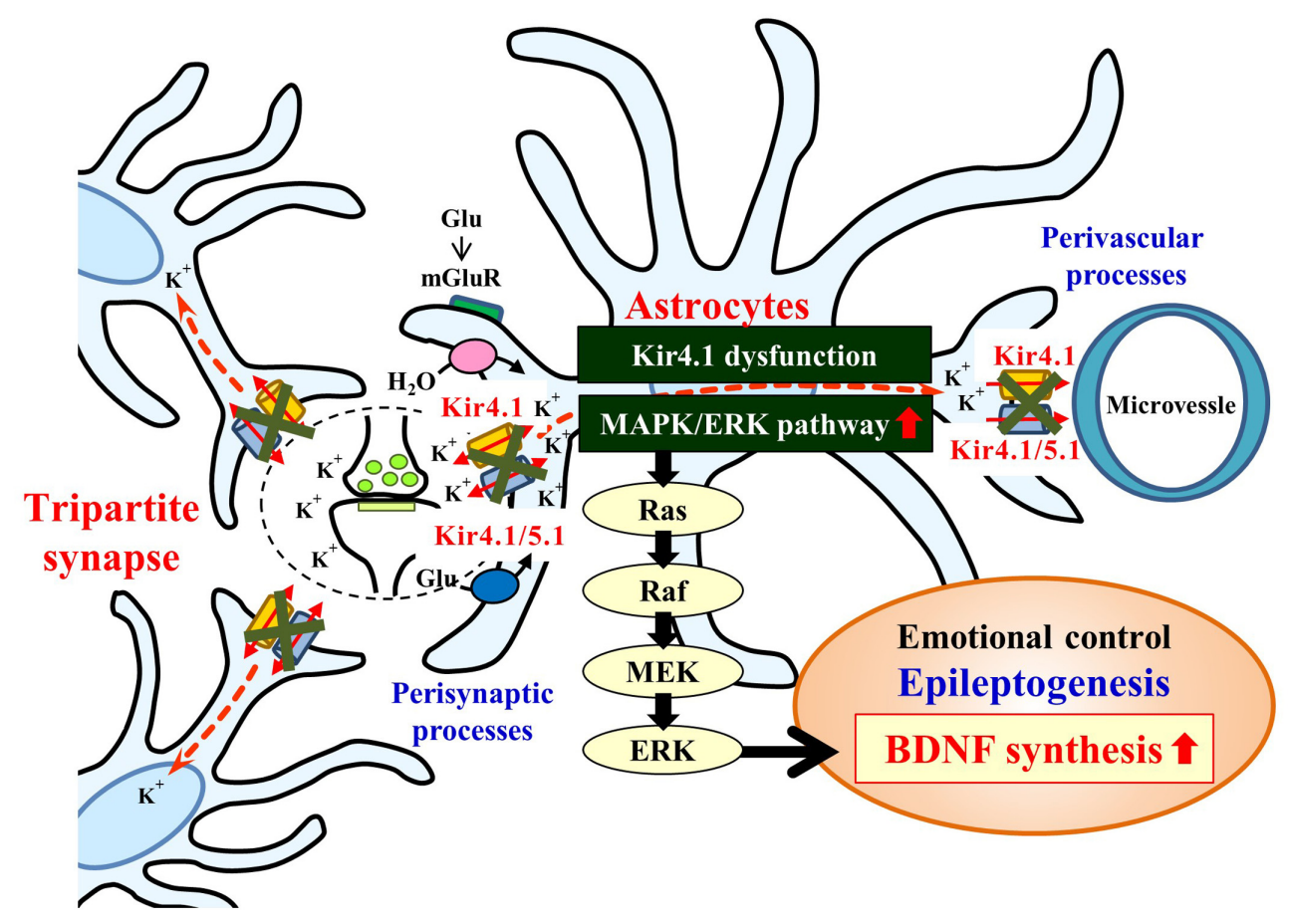

FIGURE 7 | Schematic drawing illustrating the effects of the Kir4.1 inhibition on the BDNF expression in astrocytes. Inhibition (blockade and knockdown) of Kir4.1 channels activates the Ras/Raf/MEK/ERK signaling pathway and enhances BDNF expression in astrocytes, which modulates the development of epilepsy (epileptogenesis) and other neuropsychiatric disorders (e.g., major depression).

of the KCNJ10 gene polymorphism or down-regulation of the Kir4.1 expression was observed in idiopathic generalized epilepsy patients (Phani et al., 2014; Guo et al., 2015) and TLE patients (Das et al., 2012; Heuser et al., 2012; Steinhäuser et al., 2012), respectively. The most frequent mutation of KCNJ10 in patients with EAST/SeSAME syndrome was Arg65Pro at the cytoplasmic end of the TM-1 domain. Other mutations include Thr57Ile, Arg65Cys, Phe75Leu, Gly77Arg (TM-1), Phe119fs (pore helix), Cys140Arg (extracellular loop between TM-1 and TM-2), Thr164lle, Ala167Val (cytoplasmic end of TM-2), Arg175Gln, Arg199X, Arg204His, Leu218Phe, Val259fs, and Arg297Cys (C-terminal domain) (Reichold et al., 2010; Sala-Rabanal et al., 2010; Tang et al., 2010; Freudenthal et al., 2011; Scholl et al., 2012; Kara et al., 2013; Hasan et al., 2017; Papavasiliou et al., 2017). All these mutations commonly caused suppression of Kir currents conducted by Kir4.1 channels and Kir4.1/5.1 channels, which consequently elevates the extracellular $\mathrm{K}^{+}$and glutamate levels at synapses and increases neural excitability. Besides these ictogenic influences, the present study provides evidence that inhibition of Kir4.1 channels enhances BDNF expression in astrocytes. Although the mechanisms of BDNF in facilitating epileptogenesis (the development of chronic epilepsy) are still uncertain, numerous studies have shown that BDNF expression were up-regulated in various animal models of epilepsy and in human epileptic disorders and that inhibition of BDNF/TrkB function suppressed the development of epilepsy, suggesting that BDNF plays a causal role in epilepsy induction (Kokaia et al., 1995; Binder et al., 1999; Jankowsky and Patterson, 2001; Barton and Shannon, 2005; Heinrich et al., 2011; Grabenstatter et al., 2012; Liu et al., 2013). Thus, secreted BDNF facilitate epileptogenesis possibly through acting on TrkB receptors in neurons (e.g., causing neural sprouting) (Kokaia et al., 1995; Jankowsky and Patterson, 2001; Hagihara et al., 2005). In addition, since activation of truncated TrkB-T1 receptors in astrocytes have been shown to facilitate astrogliosis, this may also be involved in epileptogenesis (Goutan et al., 1998; Climent et al., 2000).

Besides epilepsy, BDNF is implicated in the pathogenesis and treatment of neuropsychiatric disorders such as major depression (Shimizu et al., 2003; Lang et al., 2004) and autism spectrum disorder patients (Sicca et al., 2011; Sicca et al., 2016). The present finding that several antidepressants enhanced the BDNF expression by inhibiting astrocytic Kir4.1 channels leads to a hypothesis that astrocytic Kir4.1 channels can serve as a novel therapeutic target for the antidepressants (Ohno and Kurachi, 2007; Ohno et al., 2015). This possibility was supported by previous findings that antidepressants induce the BDNF expression in astrocytes (Allaman et al., 2011; HisaokaNakashima et al., 2016). In addition, involvement of the MEK signaling pathway in induction of BDNF by the Kir4.1 knockdown was consistent with the mechanisms reported in the antidepressants-induced BDNF induction (Hisaoka-Nakashima et al., 2016). Thus, the Kir4.1-BDNF system in astrocytes may be important as a non-monoaminergic mechanism underlying the 
antidepressants' action. Since pathophysiological alterations of astrocytic Kir4.1 channels were also demonstrated in Parkinson's disease (Howells et al., 2000; Ziebell et al., 2012), Alzheimer's disease (Wilcock et al., 2009), amyotrophic lateral sclerosis (Kaiser et al., 2006; Bataveljić et al., 2012), and Huntington's disease (Tong et al., 2014), further studies are required to elucidate the pathophysiological roles of the astrocytic Kir4.1-BDNF system in central nervous system disorders.

\section{CONCLUSION}

We studied the effects of Kir4.1 inhibition on expression of BDNF in primary cultured astrocytes. Blockade of Kir4.1 channels by antidepressants significantly enhanced the BDNF expression. In addition, knockdown of Kir4.1 by siRNA transfection also elevated the BDNF expression, which was preferentially suppressed by the MEK1/2 inhibitor U0126. The present results strongly suggest that inhibition of Kir4.1 channels facilitates BDNF expression in astrocytes, probably by activating the Ras/Raf/MEK/ERK pathway. Thus, the Kir4.1-BDNF system in astrocytes seems to be linked at least partly to the development of epilepsy (epileptogenesis) and other neuropsychiatric disorders. However, due to the limitations associated with experiments using cultured astrocytes, further studies are required to clarify the role and mechanisms of Kir4.1 channels in regulating BDNF expression.

\section{REFERENCES}

Allaman, I., Fiumelli, H., Magistretti, P. J., and Martin, J. L. (2011). Fluoxetine regulates the expression of neurotrophic/growth factors and glucose metabolism in astrocytes. Psychopharmacology 216, 75-84. doi: 10.1007/ s00213-011-2190-y

Araque, A., Parpura, V., Sanzgiri, R. P., and Haydon, P. G. (1999). Tripartite synapses: glia, the unacknowledged partner. Trends Neurosci. 22, 208-215.

Barton, M. E., and Shannon, H. E. (2005). The seizure-related phenotype of brainderived neurotrophic factor knockdown mice. Neuroscience 136, 563-569. doi: 10.1016/j.neuroscience.2005.08.008

Bataveljić, D., Nikolić, L., Milosević, M., Todorović, N., and Andjus, P. R. (2012). Changes in the astrocytic aquaporin-4 and inwardly rectifying potassium channel expression in the brain of the amyotrophic lateral sclerosis SOD1G93A rat model. Glia 60, 1991-2003. doi: 10.1002/glia.22414

Binder, D. K., Routbort, M. J., Ryan, T. E., Yancopoulos, G. D., and McNamara, J. O. (1999). Selective inhibition of kindling development by intraventricular administration of TrkB receptor body. J. Neurosci. 19, 1424-1436.

Bockenhauer, D., Feather, S., Stanescu, H. C., Bandulik, S., Zdebik, A. A., Reichold, M., et al. (2009). Epilepsy, ataxia, sensorineural deafness, tubulopathy, and KCNJ10 mutations. N. Engl. J. Med. 360, 1960-1970. doi: 10.1056/ NEJMoa0810276

Cheng, A., Coksaygan, T., Tang, H., Khatri, R., Balice-Gordon, R. J., Roa, M. S., et al. (2007). Truncated tyrosine kinase B brain-derived neurotrophic factor receptor directs cortical neural stem cells to a glial cell fate by a novel signaling mechanism. J. Neurochem. 100, 1515-1530. doi: 10.1111/j.1471-4159.2006. 04337.x

Climent, E., Sancho-Tello, M., Miñana, R., Barettino, D., and Guerri, C. (2000). Astrocytes in culture express the full-length Trk-B receptor and respond to brain derived neurotrophic factor by changing intracellular calcium levels: effect of ethanol exposure in rats. Neurosci. Lett. 288, 53-56. doi: 10.1016/ S0304-3940(00)01207-6

Curtis, J., and Finkbeiner, S. (1999). Sending signals from the synapse to the nucleus: possible roles for CaMK, Ras/ERK, and SAPK pathways in the

\section{AUTHOR CONTRIBUTIONS}

YO designed the research. MK, TM, YN, YM, YT, ST, KT, SS, SI, and YO performed experiments. MK, TM, SS, HI, AIk, AIn, YK, and $\mathrm{YO}$ analyzed data. MK, SS, HI, AIk, and YO wrote the paper.

\section{FUNDING}

This study was supported in part by a research grant from the Japan Agency for Medical Research and Development (YO: 15ek0109120s0701) and from a Grants-in-Aid for Scientific Research from the Ministry of Education, Culture, Sports, Science and Technology (YO: 17K08324 and 15H04892, SS:16K21501, AIk: 15H05874, HI: 15H04270).

\section{SUPPLEMENTARY MATERIAL}

The Supplementary Material for this article can be found online at: https://www.frontiersin.org/articles/10.3389/fnmol. 2017.00408/full\#supplementary-material

FIGURE S1 | Representative current traces showing inhibitory effects of fluoxetine (3-30 $\mu$ M) on Kir4.1-conducted currents in HEK293T cells. HEK293T cells expressing Kir4.1 channels were voltage-clamped at $-40 \mathrm{mV}$ and a pair of step pulses ( $\pm 70 \mathrm{mV}, 500 \mathrm{~ms}$ duration with a 200-ms interval) were applied every $20 \mathrm{~s}$. The arrowhead indicates the current level recorded at $-40 \mathrm{mV}$.

regulation of synaptic plasticity and neuronal growth. J. Neurosci. Res. 58, 88-95.

Das, A., Wallace, G. C., Holmes, C., McDowell, M. L., Smith, J. A., Marshall, J. D., et al. (2012). Hippocampal tissue of patients with refractory temporal lobe epilepsy is associated with astrocyte activation, inflammation, and altered expression of channels and receptors. Neuroscience 220, 237-246. doi: 10.1016/ j.neuroscience.2012.06.002

Devinsky, O., Vezzani, A., Najjar, S., De Lanerolle, N. C., and Rogawski, M. A. (2013). Glia and epilepsy: excitability and inflammation. Trends Neurosci. 36, 174-184. doi: 10.1016/j.tins.2012.11.008

Djukic, B., Casper, K. B., Philpot, B. D., Chin, L. S., and McCarthy, K. D. (2007). Conditional knock-out of Kir4.1 leads to glial membrane depolarization, inhibition of potassium and glutamate uptake, and enhanced short-term synaptic potentiation. J. Neurosci. 27, 11354-11365. doi: 10.1523/JNEUROSCI. 0723-07.2007

Duman, R. S., and Voleti, B. (2012). Signaling pathways underlying the pathophysiology and treatment of depression: novel mechanisms for rapid-acting agents. Trends Neurosci. 35, 47-56. doi: 10.1016/j.tins.2011. 11.004

Ferraro, T. N., Golden, G. T., Smith, G. G., Martin, J. F., Lohoff, F. W., Gieringer, T. A., et al. (2004). Fine mapping of a seizure susceptibility locus on mouse Chromosome 1: nomination of Kcnj10 as a causative gene. Mamm. Genome 15, 239-251. doi: 10.1007/s00335-003-2270-3

Freudenthal, B., Kulaveerasingam, D., Lingappa, L., Shah, M. A., Brueton, L., Wassmer, E., et al. (2011). KCNJ10 mutations disrupt function in patients with EAST syndrome. Nephron Physiol. 119, 40-48. doi: 10.1159/00033 0250

Furutani, K., Ohno, Y., Inanobe, A., Hibino, H., and Kurachi, Y. (2009). Mutational and in silico analyses for antidepressant block of astroglial inward-rectifier Kir4.1 channel. Mol. Pharmacol. 75, 1287-1295. doi: 10.1124/mol.108.052936

Goutan, E., Marti, E., and Ferrer, I. (1998). BDNF, and full length and truncated TrkB expression in the hippocampus of the rat following kainic acid excitotoxic damage. Evidence of complex time-dependent and cell-specific responses. Mol. Brain Res. 59, 154-164. doi: 10.1016/S0169-328X(98)00156-9 
Grabenstatter, H. L., Russek, S. J., and Brooks-Kayal, A. R. (2012). Molecular pathways controlling inhibitory receptor expression. Epilepsia 53, 71-78. doi: $10.1111 /$ epi.12036

Guo, Y., Yan, K. P., Qu, Q., Qu, J., Chen, Z. G., Song, T., et al. (2015). Common variants of KCNJ10 are associated with susceptibility and anti-epileptic drug resistance in Chinese genetic generalized epilepsies. PLOS ONE 10:e0124896. doi: 10.1371 /journal.pone.0124896

Hagihara, H., Hara, M., Tsunekawa, K., Nakagawa, Y., Sawada, M., and Nakano, K. (2005). Tonic-clonic seizures induce division of neuronal progenitor cells with concomitant changes in expression of neurotrophic factors in the brain of pilocarpine-treated mice. Brain Res. Mol. Brain Res. 139, 258-266. doi: 10.1016/ j.molbrainres.2005.05.031

Harada, Y., Nagao, Y., Shimizu, S., Serikawa, T., Terada, R., Fujimoto, M., et al. (2013). Expressional analysis of inwardly rectifying Kir4.1 channels in Noda epileptic rat (NER). Brain Res. 1517, 141-149. doi: 10.1016/j.brainres.2013. 04.009

Hasan, S., Balobaid, A., Grottesi, A., Dabbagh, O., Cenciarini, M., Rawashdeh, R., et al. (2017). Lethal digenic mutations in the $\mathrm{K}+$ channels Kir4.1 (KCNJ10) and SLACK (KCNT1) associated with severe-disabling seizures and neurodevelopmental delay. J. Neurophysiol. 118, 2402-2411. doi: 10.1152/jn. 00284.2017

Heinrich, C., Lähteinen, S., Suzuki, F., Anne-Marie, L., Huber, S., Häussler, U., et al. (2011). Increase in BDNF-mediated TrkB signaling promotes epileptogenesis in a mouse model of mesial temporal lobe epilepsy. Neurobiol. Dis. 42, 35-47. doi: 10.1016/j.nbd.2011.01.001

Heuser, K., Eid, T., Lauritzen, F., Thoren, A. E., Vindedal, G. F., Taubøll, E., et al. (2012). Loss of perivascular Kir4.1 potassium channels in the sclerotic hippocampus of patients with mesial temporal lobe epilepsy. J. Neuropathol. Exp. Neurol. 71, 814-825. doi: 10.1097/NEN.0b013e318267b5af

Heuser, K., Nagelhus, E. A., Taubøll, E., Indahl, U., Berg, P. R., Lien, S., et al. (2010). Variants of the genes encoding AQP4 and Kir4.1 are associated with subgroups of patients with temporal lobe epilepsy. Epilepsy Res. 88, 55-64. doi: 10.1016/j.eplepsyres.2009.09.023

Hisaoka-Nakashima, K., Kajitani, N., Kaneko, M., Shigetou, T., Kasai, M., Matsumoto, C., et al. (2016). Amitriptyline induces brain-derived neurotrophic factor (BDNF) mRNA expression through ERK-dependent modulation of multiple BDNF mRNA variants in primary cultured rat cortical astrocytes and microglia. Brain Res. 1634, 57-67. doi: 10.1016/j.brainres.2015.12.057

Howells, D. W., Porritt, M. J., Wong, J. Y., Batchelor, P. E., Kalnins, R., Hughes, A. J., et al. (2000). Reduced BDNF mRNA expression in the Parkinson's disease substantia nigra. Exp. Neurol. 166, 127-135. doi: 10.1006/exnr.2000.7483

Inyushin, M., Kucheryavykh, L. Y., Kucheryavykh, Y. V., Nichols, C. G., Buono, R. J., Ferraro, T. N., et al. (2010). Potassium channel activity and glutamate uptake are impaired in astrocytes of seizure-susceptible DBA/2 mice. Epilepsia 51, 1707-1713. doi: 10.1111/j.1528-1167.2010.02592.x

Jankowsky, J. L., and Patterson, P. H. (2001). The role of cytokines and growth factors in seizures and their sequelae. Prog. Neurobiol. 63, 125-149.

Jean, Y. Y., Lercher, L. D., and Dreyfus, C. F. (2008). Glutamate elicits release of BDNF from basal forebrain astrocytes in a process dependent on metabotropic receptors and the PLC pathway. Neuron Glia Biol. 4, 35-42. doi: 10.1017/ S1740925X09000052

Kaiser, M., Maletzki, I., Hülsmann, S., Holtmann, B., Schulz-Schaeffer, W., Kirchhoff, F., et al. (2006). Progressive loss of a glial potassium channel $(K C N J 10)$ in the spinal cord of the SOD1 (G93A) transgenic mouse model of amyotrophic lateral sclerosis. J. Neurochem. 99, 900-912. doi: 10.1111/j.14714159.2006.04131.x

Kara, B., Ekici, B., Ipekçi, B., Aslanger, A. K., and Scholl, U. (2013). KCNJ10 gene mutation in an 8-year-old boy with seizures. Acta Neurol. Belg. 113, 75-77. doi: 10.1007/s13760-012-0113-2

Kofuji, P., Ceelen, P., Zahs, K. R., Surbeck, L. W., Lester, H. A., and Newman, E. A. (2000). Genetic inactivation of an inwardly rectifying potassium channel (Kir4.1 subunit) in mice: phenotypic impact in retina. J. Neurosci. 20, 5733-5740.

Kofuji, P., and Newman, E. A. (2004). Potassium buffering in the central nervous system. Neuroscience 129, 1045-1056. doi: 10.1016/j.neuroscience.2004.06.008

Kokaia, M., Ernfors, P., Kokaia, Z., Elmér, E., Jaenisch, R., and Lindvall, O. (1995). Suppressed epileptogenesis in BDNF mutant mice. Exp. Neurol. 133, 215-224. doi: 10.1006/exnr.1995.1024
Kong, E. K., Peng, L., Chen, Y., Yu, A. C., and Hertz, L. (2002). Up-regulation of 5-HT2B receptor density and receptor-mediated glycogenolysis in mouse astrocytes by long-term fluoxetine administration. Neurochem. Res. 27, $113-120$.

Lang, U. E., Hellweg, R., and Gallinat, J. (2004). BDNF serum concentrations in healthy volunteers are associated with depression-related personality traits. Neuropsychopharmacology 29, 795-798. doi: 10.1038/sj.npp.1300382

Li, B., Dong, L., Wang, B., Cai, L., Jiang, N., and Peng, L. (2012). Cell typespecific gene expression and editing responses to chronic fluoxetine treatment in the in vivo mouse brain and their relevance for stress-induced anhedonia. Neurochem. Res. 37, 2480-2495. doi: 10.1007/s11064-012-0814-1

Liu, G., Gu, B., He, X. P., Joshi, R. B., Wackerle, H. D., Rodriguiz, R. M., et al. (2013). Transient inhibition of TrkB kinase following status epilepticus prevents development of temporal lobe epilepsy. Neuron 79, 31-38. doi: 10.1016/j. neuron.2013.04.027

Mortensen, O. V., Kristensen, A. S., and Wiborg, O. (2001). Species-scanning mutagenesis of the serotonin transporter reveals residues essential in selective, high-affinity recognition of antidepressants. J. Neurochem. 79, 237-247.

Nagao, Y., Harada, Y., Mukai, T., Shimizu, S., Okuda, A., Fujimoto, M., et al. (2013). Expressional analysis of the astrocytic Kir4.1 channel in a pilocarpine-induced temporal lobe epilepsy model. Front. Cell. Neurosci. 7:104. doi: 10.3389/fncel. 2013.00104

Neusch, C., Papadopoulos, N., Müller, M., Maletzki, I., Winter, S. M., Hirrlinger, J., et al. (2006). Lack of the Kir4.1 channel subunit abolishes $\mathrm{K}+$ buffering properties of astrocytes in the ventral respiratory group: impact on extracellular K+ regulation. J. Neurophysiol. 95, 1843-1852. doi: 10.1152/jn.00996.2005

Neusch, C., Rozengurt, N., Jacobs, R. E., Lester, H. A., and Kofuji, P. (2001). Kir4.1 potassium channel subunit Is crucial for oligodendrocyte development and in vivo myelination. J. Neurosci. 21, 5429-5438.

Ohno, Y., Hibino, H., Lossin, C., Inanobe, A., and Kurachi, Y. (2007). Inhibition of astroglial Kir4.1 channels by selective serotonin reuptake inhibitors. Brain Res. 1178, 44-51. doi: 10.1016/j.brainres.2007.08.018

Ohno, Y., Ishihara, S., Terada, R., Kikuta, M., Sofue, N., Kawai, Y., et al. (2009). Preferential increase in the hippocampal synaptic vesicle protein 2A (SV2A) by pentylenetetrazole kindling. Biochem. Biophys. Res. Commun. 390, 415-420. doi: 10.1016/j.bbrc.2009.09.035

Ohno, Y., and Kurachi, Y. (2007). "Astroglial inwardly rectifying potassium channel Kir4.1 as a potential target for the novel antidepressant agents," in Drug Design Research Perspectives, ed. S. P. Kaplan (Hauppauge, NY: Nova Science Publishers, Inc.), 1-8.

Ohno, Y., Tokudome, K., Kunisawa, N., Iha, H. A., Kinboshi, M., Mukai, T., et al. (2015). Role of astroglial Kir4.1 channels in the pathogenesis and treatment of epilepsy. Ther. Targets Neurol. Dis. 2:e476. doi: 10.14800/ttnd.476

Olsen, M. L., and Sontheimer, H. (2008). Functional implications for Kir4.1 channels in glia biology: from $\mathrm{K}+$ buffering to cell differentiation. J. Neurochem. 107, 589-601. doi: 10.1111/j.1471-4159.2008.05615.x

Papavasiliou, A., Foska, K., Ioannou, J., and Nagel, M. (2017). Epilepsy, ataxia, sensorineural deafness, tubulopathy syndrome in a European child with KCNJ10 mutations: a case report. SAGE Open Med. Case Rep. 5, 1-6. doi: $10.1177 / 2050313 \times 17723549$

Parpura, V., and Zorec, R. (2010). Gliotransmission: exocytotic release from astrocytes. Brain Res. Rev. 63, 83-92. doi: 10.1016/j.brainresrev.2009.11.008

Phani, N. M., Acharya, S., Xavy, S., Bhaskaranand, N., Bhat, M. K., Jain, A., et al. (2014). Genetic association of KCNJ10 rs1130183 with seizure susceptibility and computational analysis of deleterious non-synonymous SNPs of KCNJ10 gene. Gene 536, 247-253. doi: 10.1016/j.gene.2013.12.026

Quesseveur, G., David, D. J., Gaillard, M. C., Pla, P., Wu, M. V., Nguyen, H. T., et al. (2013). BDNF overexpression in mouse hippocampal astrocytes promotes local neurogenesis and elicits anxiolytic-like activities. Transl. Psychiatry 3:e253. doi: $10.1038 /$ tp.2013.30

Reichold, M., Zdebik, A. A., Lieberer, E., Rapedius, M., Schmidt, K., Bandulik, S., et al. (2010). KCNJ10 gene mutations causing EAST syndrome (epilepsy, ataxia, sensorineural deafness, and tubulopathy) disrupt channel function. Proc. Natl. Acad. Sci. U.S.A. 107, 14490-14495. doi: 10.1073/pnas.1003072107

Rose, C. R., Blum, R., Pichler, B., Lepier, A., Kafitz, K. W., and Konnerth, A. (2003). Truncated TrkB-T1 mediates neurotrophin-evoked calcium signalling in glia cells. Nature 426, 74-78. doi: 10.1038/nature01983 
Sala-Rabanal, M., Kucheryavykh, L. Y., Skatchkov, S. N., Eaton, M. J., and Nichols, C. G. (2010). Molecular mechanisms of EAST/SeSAME syndrome mutations in Kir4.1 (KCNJ10). J. Biol. Chem. 285, 36040-36048. doi: 10.1074/jbc.M110. 163170

Scholl, U. I., Choi, M., Liu, T., Ramaekers, V. T., Häusler, M. G., Grimmer, J., et al. (2009). Seizures, sensorineural deafness, ataxia, mental retardation, and electrolyte imbalance (SeSAME syndrome) caused by mutations in KCNJ10. Proc. Natl. Acad. Sci. U.S.A. 106, 5842-5847. doi: 10.1073/pnas.0901749106

Scholl, U. I., Dave, H. B., Lu, M., Farhi, A., Nelson-Williams, C., Listman, J. A., et al. (2012). SeSAME/EAST syndrome-phenotypic variability and delayed activity of the distal convoluted tubule. Pediatr. Nephrol. 27, 2081-2090. doi: 10.1007/ s00467-012-2219-4

Shimizu, E., Hashimoto, K., Okamura, N., Koike, K., Komatsu, N., Kumakiri, C., et al. (2003). Alterations of serum levels of brain-derived neurotrophic factor (BDNF) in depressed patients with or without antidepressants. Biol. Psychiatry $54,70-75$.

Sicca, F., Ambrosini, E., Marchese, M., Sforna, L., Servettini, I., Valvo, G., et al. (2016). Gain-of-function defects of astrocytic Kir4.1 channels in children with autism spectrum disorders and epilepsy. Sci. Rep. 6:34325. doi: 10.1038/ srep34325

Sicca, F., Imbrici, P., D’Adamo, M. C., Moro, F., Bonatti, F., Brovedani, P., et al. (2011). Autism with seizures and intellectual disability: possible causative role of gain-of-function of the inwardly-rectifying K+ channel Kir4.1. Neurobiol. Dis. 43, 239-247. doi: 10.1016/j.nbd.2011.03.016

Simard, M., and Nedergaard, M. (2004). The neurobiology of glia in the context of water and ion homeostasis. Neuroscience 129, 877-896. doi: 10.1016/j. neuroscience.2004.09.053

Steinhäuser, C., Seifert, G., and Bedner, P. (2012). Astrocyte dysfunction in temporal lobe epilepsy: $\mathrm{K}+$ channels and gap junction coupling. Glia 60 , 1192-1202. doi: 10.1002/glia.22313

Su, S., Ohno, Y., Lossin, C., Hibino, H., Inanobe, A., and Kurachi, Y. (2007). Inhibition of astroglial inwardly rectifying Kir4.1 channels by a tricyclic antidepressant, nortriptyline. J. Pharmacol. Exp. Ther. 320, 573-580. doi: 10. 1124/jpet.106.112094

Tang, X., Hang, D., Sand, A., and Kofuji, P. (2010). Variable loss of Kir4.1 channel function in SeSAME syndrome mutations. Biochem. Biophys. Res. Commun. 399, 537-541. doi: 10.1016/j.bbrc.2010.07.105

Tong, X., Ao, Y., Faas, G. C., Nwaobi, S. E., Xu, J., Haustein, M. D., et al. (2014). Astrocyte Kir4.1 ion channel deficits contribute to neuronal dysfunction in Huntington's disease model mice. Nat. Neurosci. 17, 694-703. doi: 10.1038/nn. 3691

Walz, W. (2000). Role of astrocytes in the clearance of excess extracellular potassium. Neurochem. Int. 36, 291-300.

Wilcock, D. M., Vitek, M. P., and Colton, C. A. (2009). Vascular amyloid alters astrocytic water and potassium channels in mouse models and humans with Alzheimer's disease. Neuroscience 159, 1055-1069. doi: 10.1016/j.neuroscience. 2009.01.023

Ziebell, M., Khalid, U., Klein, A. B., Azar, S., Thomsen, G., Jansen, P., et al. (2012). Striatal dopamine transporter binding correlates with serum BDNF levels in patients with striatal dopaminergic neurodegeneration. Neurobiol. Aging 33, 428.e1-5. doi: 10.1016/j.neurobiolaging.2010. 11.010

Conflict of Interest Statement: The authors declare that the research was conducted in the absence of any commercial or financial relationships that could be construed as a potential conflict of interest.

Copyright (๑ 2017 Kinboshi, Mukai, Nagao, Matsuba, Tsuji, Tanaka, Tokudome, Shimizu, Ito, Ikeda, Inanobe, Kurachi, Inoue and Ohno. This is an open-access article distributed under the terms of the Creative Commons Attribution License (CC BY). The use, distribution or reproduction in other forums is permitted, provided the original author(s) or licensor are credited and that the original publication in this journal is cited, in accordance with accepted academic practice. No use, distribution or reproduction is permitted which does not comply with these terms. 